\title{
THE STRUCTURE OF GROUPS WHOSE SUBGROUPS ARE PERMUTABLE-BY-FINITE
}

\author{
M. DE FALCO, F. DE GIOVANNI ${ }^{\circledR}$, C. MUSELLA and Y. P. SYSAK
}

(Received 22 October 2004; revised 21 March 2005)

Communicated by E. A. O'Brien

\begin{abstract}
A subgroup $H$ of a group $G$ is said to be permutable if $H X=X H$ for each subgroup $X$ of $G$, and the group $G$ is called quasihamiltonian if all its subgroups are permutable. We shall say that $G$ is a $Q F$-group if every subgroup $H$ of $G$ contains a subgroup $K$ of finite index which is permutable in $G$. It is proved that every locally finite $Q F$-group contains a quasihamiltonian subgroup of finite index. In the proof of this result we use a theorem by Buckley, Lennox, Neumann, Smith and Wiegold concerning the corresponding problem when permutable subgroups are replaced by normal subgroups: if $G$ is a locally finite group such that $H / H_{G}$ is finite for every subgroup $H$, then $G$ contains an abelian subgroup of finite index.
\end{abstract}

2000 Mathematics subject classification: primary $20 \mathrm{E} 15$.

\section{Introduction}

A subgroup $X$ of a group $G$ is said to be permutable if $X H=H X$ for every subgroup $H$ of $G$. This concept was introduced by Ore, and the behaviour of permutable subgroups was later investigated by several authors. A group is called quasihamiltonian if all its subgroups are permutable. It was proved by Stonehewer [12] that permutable subgroups of arbitrary groups are ascendant, so that quasihamiltonian groups are locally nilpotent. The structure of quasihamiltonian groups was described by Iwasawa $([6,7])$. In particular, quasihamiltonian primary groups are either abelian or contain an abelian subgroup of finite index and finite exponent. For a detailed account of results concerning permutable subgroups we refer to [11, Chapters 5 and 6 ].

(c) 2006 Australian Mathematical Society $1446-7887 / 06 \$ A 2.00+0.00$ 
Some classical theorems of Neumann [9] deal with groups in which every subgroup is close to being normal in a suitable sense. In particular, Neumann described groups in which every subgroup is normal in a subgroup of finite index and those in which all subgroups have finite index in their normal closures; corresponding results to these theorems for generalized permutable subgroups have been obtained in [2] and [5], respectively. Recently, the structure of groups in which all subgroups are normal-byfinite has been investigated; here a subgroup $H$ of a group $G$ is said to be normal-byfinite if the core $H_{G}$ of $H$ in $G$ has finite index in $H$, and $G$ is called a $C F$-group if all its subgroups are normal-by-finite. It has been proved in [1] that every locally finite $C F$-group is abelian-by-finite. The aim of this paper is to extend this latter theorem, replacing normality by permutability.

We shall say that a subgroup $H$ of a group $G$ is permutable-by-finite if it contains a permutable subgroup $K$ of $G$ such that the index $|H: K|$ is finite; the group $G$ is called a $Q F$-group if all its subgroups are permutable-by-finite. Our result here is the following.

THEOREM. Let $G$ be a locally finite group in which every subgroup is permutableby-finite. Then $G$ contains a quasihamiltonian subgroup of finite index.

Consideration of any Tarski group shows that the local finiteness hypothesis cannot be omitted in this theorem.

Most of our notation is standard and can be found in [10]. For any subgroup $H$ of a group $G$, we denote by $H_{*}$ the subgroup of $H$ generated by all permutable subgroups of $G$ contained in $H$. Since the conjugates and the join of any collection of permutable subgroups are likewise permutable, the subgroup $H_{*}$ is normal in $H$ and permutable in $G$; it will be called the permutable core of $H$ in $G$.

\section{Some preliminary results}

In this short section we collect some general results that will be useful in the proof of our main theorem.

We say that a group $G$ is an iterated semidirect product of a sequence $\left(K_{n}\right)_{n \in \gg}$ of its subgroups if the following conditions are satisfied:

(i) $G=\left\langle K_{n} \mid n \in \mathbb{N}\right\rangle$;

(ii) the subgroup $K_{n+1}$ is normalized by $K_{1}, \ldots, K_{n}$ for each positive integer $n$;

(iii) $\left\langle K_{1}, \ldots, K_{n}\right\rangle \cap K_{n+1}=\{1\}$ for all $n$.

Our first lemma shows that iterated semidirect products of infinitely many nonabelian subgroups cannot occur in the structure of abelian-by-finite groups. 
LEMMA 2.1. Let the group $G$ be an iterated semidirect product of infinitely many non-abelian subgroups $K_{1}, \ldots, K_{n}, K_{n+1}, \ldots$ Then $G$ is not abelian-by-finite.

ProOF. Assume for a contradiction that the lemma is false, and consider a counterexample $G$ containing an abelian normal subgroup $A$ with smallest possible index. Clearly, $G$ has infinite derived subgroup, so that $A$ cannot be contained in the centre of $G$ by Schur's theorem (see for instance [10, Part 1, Theorem 4.12]). For each positive integer $n$, put $L_{n}=\left\langle K_{1}, \ldots, K_{n}\right\rangle$, so that

$$
G=\bigcup_{n \in \mathbb{N}} L_{n} \quad \text { and } \quad A=\bigcup_{n \in \mathbb{N}}\left(A \cap L_{n}\right) .
$$

Therefore there exists some $m \geq 1$ such that $G=A L_{m}$ and $A \cap L_{m}$ is a non-central subgroup of $G$. In particular, the centralizer $C_{G}\left(A \cap L_{m}\right)$ is a proper subgroup of $G$ containing $A$. On the other hand, $A \cap L_{m}$ is a normal subgroup of $G$ which normalizes and so centralizes every subgroup $K_{n}$ with $n \geq m+1$. Thus $C_{G}\left(A \cap L_{m}\right)$ contains the subgroup

$$
H=\left\langle K_{n} \mid n \geq m+1\right\rangle,
$$

and hence

$$
|H: A \cap H| \leq\left|C_{G}\left(A \cap L_{m}\right): A\right|<|G: A| .
$$

As $H$ is the iterated semidirect product of its non-abelian subgroups $K_{m+1}, K_{m+2}, \ldots$, this contradicts the minimal choice of $G$.

If $\mathfrak{X}$ is a class of groups, the $\mathfrak{X}$-residual of a group $G$ is the intersection of all normal subgroups $N$ of $G$ such that $G / N$ belongs to $\mathfrak{X}$, and $G$ is called a residually $\mathfrak{X}$-group if it has trivial $\mathfrak{X}$-residual. Clearly, the $\mathfrak{X}$-residual of any group $G$ is the smallest normal subgroup $J$ of $G$ such that the factor group $G / J$ is residually $\mathfrak{X}$. The proof of the following result is straightforward.

LEMMA 2.2. Let $G$ be a residually (central-by-finite) group. Then the factor group $G / Z(G)$ is residually finite.

Recall that if $H$ is a subgroup of a group $G$, the profinite closure of $H$ in $G$ is the intersection of all subgroups of finite index of $G$ containing $H$.

LEMMA 2.3. Let $G$ be a periodic residually finite group, and let $H$ be a subgroup of $G$ with finite exponent $e$. Then the profinite closure $\hat{H}$ of $H$ in $G$ has exponent $e$.

PROOF. Let $\left(G_{i}\right)_{i \in I}$ be the collection of all normal subgroups of finite index of $G$. Then $\hat{H}=\bigcap_{i \in I} H G_{i}$, and hence for every $h \in \hat{H}$ and for each $i \in I$, there exist 
elements $h_{i} \in H$ and $g_{i} \in G_{i}$ such that $h=h_{i} g_{i}$. Since

$$
h^{e}=h_{i}^{e} g_{i}^{h_{i}^{e-1}} \cdots g_{i}^{h_{i}} g_{i}=g_{i}^{h_{i}^{e-1}} \cdots g_{i}^{h_{i}} g_{i}
$$

belongs to $G_{i}$ for all $i \in I$, it follows that $h^{e}=1$, and $\hat{H}$ has exponent $e$.

\section{Primary groups}

The aim of this section is to prove that primary locally finite $Q F$-groups are abelianby-finite. The case of metabelian groups plays a central role in our considerations.

LEMMA 3.1. Let $G$ be a metabelian p-group with the property $Q F$. If the derived subgroup $G^{\prime}$ of $G$ has finite exponent, then all subgroups of $G$ are subnormal.

ProOF. If $x$ is any element of $G$, the normal subgroup $\left\langle x, G^{\prime}\right\rangle$ is nilpotent (see [10, Part 2, Lemma 6.34]), and so $G$ is a Fitting group. Let $X$ be any subgroup of $G$, and let $X_{*}$ be the permutable core of $X$ in $G$. Then $X=X_{*} E$, where $E$ is a suitable finite subgroup of $G$. Since $X_{*}$ is subnormal in $G$ (see [4, Lemma 2.2]), it follows that $X$ is subnormal in $G$.

LEMMA 3.2. Let $G$ be a metabelian p-group with the property $Q F$. If the derived subgroup $G^{\prime}$ of $G$ has finite exponent, then $G$ is abelian-by-finite.

Proof. Suppose first that $G^{\prime}$ has exponent $p$, and let $E$ be a finite non-abelian subgroup of $G$. Assume that the group $E G^{\prime} / Z\left(E G^{\prime}\right)$ is infinite, so that

$$
G^{\prime}=C_{G^{\prime}}(E) \times K,
$$

where $K$ is an infinite subgroup. Then the permutable core $K_{*}$ of $K$ in $G$ is also infinite. Clearly, $K_{*}$ has finite index in $E K_{*}$, and so it contains a subgroup of finite index $L$ such that $L^{E}=L$. As the group $E L$ is nilpotent (see [10, Part 2, Lemma 6.34]), we obtain that $C_{L}(E) \neq\{1\}$. This contradiction shows that the normal subgroup $E G^{\prime}$ of $G$ is central-by-finite, so that by Schur's theorem its derived subgroup is a finite non-trivial normal subgroup of $G$ (see [10, Part 1, Theorem 4.12]). It follows that $G$ is hypercentral. Moreover, all subgroups of $G$ are subnormal by Lemma 3.1, and hence $G$ is nilpotent by a result of Möhres [8]. Therefore $G$ is abelian-by-finite (see [4, Lemma 4.2]).

Suppose now that $G^{\prime}$ has exponent $p^{n}$ with $n>1$. The above case yields that $G /\left(G^{\prime}\right)^{p}$ contains an abelian subgroup of finite index $H /\left(G^{\prime}\right)^{p}$. Thus $H^{\prime} \leq\left(G^{\prime}\right)^{p}$ has exponent at most $p^{n-1}$, and by induction on the exponent of $G^{\prime}$ we have that the $Q F$-group $H$ is abelian-by-finite. Therefore $G$ itself is abelian-by-finite. 
If $G$ is any p-group, we put $\Omega_{0}(G)=\{1\}$ and for each non-negative integer $n$, we define inductively the normal subgroup $\Omega_{n}(G)$ of $G$ by choosing $\Omega_{n+1}(G) / \Omega_{n}(G)$ as the subgroup generated by all elements of order $p$ of $G / \Omega_{n}(G)$.

LEMMA 3.3. Let $G$ be a metabelian p-group whose derived subgroup $G^{\prime}$ is residually finite and has infinite exponent. If $G$ is a $Q F$-group and the factor groups $\Omega_{n+1}(G) / \Omega_{n}(G)$ are abelian for all $n \geq 0$, then the normal closure $E^{G}$ of every finite subgroup $E$ of $G$ is central-by-finite.

PROOF. Clearly, the subgroup $E G^{\prime}$ is abelian-by-finite, and hence it is a $C F$-group (see [4, Lemma 4.1]). Thus there exists a subgroup $A$ of finite index in $G^{\prime}$ such that $\left|E: C_{E}(A)\right| \leq 2$ and every subgroup of $A$ is normal in $E G^{\prime}$ (see [1, Lemma 2.1 and Lemma 3.8]). Assume that $\left|E: C_{E}(A)\right|=2$, so that there exists $g \in E$ such that $g^{-1} a g=a^{-1}$ and so $(g a)^{2}=g^{2}$ for all $a \in A$. If the element $g$ has order $2^{n}$, it follows that $A$ is contained in $\Omega_{n}(G)$, contrary to the hypothesis. Thus $C_{E}(A)=E$, and hence the subgroup $E G^{\prime}$ is central-by-finite. As $E^{G}$ is contained in $E G^{\prime}$, the lemma is proved.

Our next result allows us to apply the above lemma in the case of residually finite $Q F$-groups.

LEMMA 3.4. Let $G$ be a locally finite p-group with the property $Q F$. If $G$ is residually finite, then there exists a normal subgroup $H$ of finite index in $G$ such that the factor groups $\Omega_{n+1}(H) / \Omega_{n}(H)$ are abelian for all $n \geq 0$.

Proof. Assume that the statement is false. We shall prove that there exist in $G$ a sequence $\left(K_{n}\right)_{n \in \mathbb{N}}$ of finite non-abelian subgroups and a descending series

$$
G=G_{0}>G_{1}>\cdots>G_{n}>\cdots
$$

of normal subgroups of finite index such that for each $n \geq 1$ the following conditions hold:

(a) if $s_{n}$ is the smallest non-negative integer such that the factor group

$$
\Omega_{s_{n}+1}\left(G_{n-1}\right) / \Omega_{s_{n}}\left(G_{n-1}\right)
$$

is non-abelian, then $K_{n} \leq \Omega_{s_{n}+1}\left(G_{n-1}\right)$ and $K_{n} /\left(K_{n} \cap \Omega_{s_{n}}\left(G_{n-1}\right)\right)$ is generated by non-permutable subgroups of order $p$;

(b) $K_{n}$ is normal in the subgroup $L_{n}=\left\langle K_{1}, \ldots, K_{n}\right\rangle$;

(c) $G_{n} \cap L_{n} \leq \Omega_{s_{n}}\left(G_{n-1}\right)$. 
In fact, let $s_{1}$ be the smallest non-negative integer such that the group $\Omega_{s_{1}+1}(G) / \Omega_{s_{1}}(G)$ is non-abelian, and let $x$ and $y$ be elements of $G$ such that each of the cosets $x \Omega_{s_{1}}(G)$ and $y \Omega_{s_{1}}(G)$ generates a non-permutable subgroup of order $p$ of $G / \Omega_{s_{1}}(G)$. Put $K_{1}=\langle x, y\rangle$, and suppose that for some $n \geq 1$ the subgroups $K_{1}, \ldots, K_{n}$ and $G_{1}, \ldots, G_{n-1}$ have been chosen. Clearly, the subgroup $\Omega_{s_{n}}\left(G_{n-1}\right)$ has exponent $p^{s_{n}}$, and so by Lemma 2.3 it coincides with its profinite closure in $G_{n-1}$. Thus the factor group $G_{n-1} / \Omega_{s_{n}}\left(G_{n-1}\right)$ is residually finite, and hence there exists a normal subgroup $G_{n}$ of finite index in $G$ such that

$$
\Omega_{s_{n}}\left(G_{n-1}\right) \leq G_{n}<G_{n-1}
$$

and

$$
G_{n} \cap L_{n} \leq \Omega_{s_{n}}\left(G_{n-1}\right) .
$$

Let $s_{n+1}$ be the least non-negative integer such that $\Omega_{s_{n+1}+1}\left(G_{n}\right) / \Omega_{s_{n+1}}\left(G_{n}\right)$ is nonabelian. Then there exists a finite subgroup $K$ of $\Omega_{s_{n+1}+1}\left(G_{n}\right)$ such that the group $K / K \cap \Omega_{s_{n+1}}\left(G_{n}\right)$ is generated by non-permutable subgroups of order $p$. Put $K_{n+1}=$ $K^{L_{n}}$. Then $K_{n+1} \leq \Omega_{s_{n+1}+1}\left(G_{n}\right)$ and $K_{n+1} /\left(K_{n+1} \cap \Omega_{s_{n+1}}\left(G_{n}\right)\right)$ is likewise generated by non-permutable subgroups of order $p$. This completes the construction of the subgroups $K_{n+1}$ and $G_{n}$.

Clearly, $s_{n} \leq s_{n+1}$ for each $n$, so that $\Omega_{s_{n}}\left(G_{n-1}\right)=\Omega_{s_{n}}\left(G_{n}\right) \leq \Omega_{s_{n+1}}\left(G_{n}\right)$, and hence

$$
N=\bigcup_{n \in \mathbb{N}} \Omega_{s_{n}}\left(G_{n-1}\right)
$$

is a normal subgroup of $G$. Moreover, since $\Omega_{s_{n+1}}\left(G_{n}\right) \leq \Omega_{s_{m+1}}\left(G_{m}\right) \leq G_{m}$ for all $m \geq n$, it follows that $N$ is contained in any $G_{n}$. As

$$
K_{n} \cap \Omega_{s_{n}}\left(G_{n-1}\right) \leq K_{n} \cap N \leq L_{n} \cap G_{n} \leq \Omega_{s_{n}}\left(G_{n-1}\right),
$$

we have that $K_{n} \cap N=K_{n} \cap \Omega_{s_{n}}\left(G_{n-1}\right)$ and hence $K_{n} / K_{n} \cap N$ is generated by non-permutable subgroups of order $p$. Finally, the relation

$$
K_{n+1} \leq \Omega_{s_{n+1}+1}\left(G_{n}\right) \leq G_{n}
$$

yields that

$$
K_{n+1} N \cap L_{n} \leq G_{n} \cap L_{n} \leq \Omega_{s_{n}}\left(G_{n-1}\right) \leq N .
$$

Consider in $G$ the subgroup $L=\bigcup_{n \in N} L_{n}$. Then the factor group $L N / N$ is the iterated semidirect product of its non-abelian subgroups $K_{1} N / N, \ldots, K_{n} N / N, \ldots$, and in particular $L N / N$ cannot be abelian-by-finite by Lemma 2.1. On the other hand, $L N / N$ is generated by elements of order $p$, so that all its permutable subgroups are normal and $L N / N$ is a $C F$-group. Thus $L N / N$ is abelian-by-finite (see [1]) and this contradiction completes the proof. 
LEMMA 3.5. Let $G$ be a metabelian p-group with the property $Q F$. If the derived subgroup $G^{\prime}$ of $G$ is residually finite, then the factor group $G / Z(G)$ is also residually finite.

Proof. Let $H$ be any subgroup of finite index of $G^{\prime}$. Then $G^{\prime} / H_{G}$ has finite exponent, so that $G / H_{G}$ is abelian-by-finite by Lemma 3.2, and hence it also has the property $C F$ (see [4, Lemma 4.1]). Thus $H / H_{G}$ is finite and $G / H_{G}$ is finite-byabelian, so that $G / H_{G}$ is even central-by-finite. As $G^{\prime}$ is residually finite, it follows that $G$ is residually (central-by-finite), and hence $G / Z(G)$ is residually finite by Lemma 2.2 .

The condition $Q F$ is essential in the above lemma, as the consideration of the standard wreath product of a group of order $p$ with a group of type $p^{\infty}$ shows.

Recall that the $F C$-centre of a group $G$ is the subgroup consisting of all elements of $G$ with finitely many conjugates, and $G$ is called an $F C$ - group if it coincides with its $F C$-centre.

LEMMA 3.6. Let $G$ be a residually finite $p$-group with the property $Q F$. If the normal closure of every finite subgroup of $G$ has finite exponent, then there exists a subgroup of finite index in $G$ whose derived subgroup has finite exponent.

Proof. Assume that the statement is false. We shall prove that there exist in $G$ a sequence $\left(K_{n}\right)_{n \in \mathbb{N}}$ of finite non-abelian subgroups and a descending series

$$
G=G_{0}>G_{1}>\cdots>G_{n}>\cdots
$$

of normal subgroups of finite index such that for each $n$ the following conditions hold:

(a) $K_{n}<G_{n-1}$;

(b) if $H_{0}=\{1\}$ and $H_{n}$ is the profinite closure of $K_{n}^{G} H_{n-1} \cap G_{n}$ in $G$, then the factor group $K_{n} /\left(H_{n-1} \cap K_{n}\right)$ is non-abelian;

(c) $L_{n} \cap G_{n} \leq H_{n-1}$, where $L_{n}=\left\langle K_{1}, \ldots, K_{n}\right\rangle$.

In fact, let $K_{1}$ be a finite non-abelian subgroup of $G$, and let $G_{1}$ be a normal subgroup of finite index of $G$ such that $K_{1} \cap G_{1}=\{1\}$. Suppose that for some $n \geq 1$ the subgroups $K_{1}, \ldots, K_{n}$ and $G_{1}, \ldots, G_{n}$ have been chosen. As the normal closure $K_{n}^{G}$ has finite exponent, it follows from Lemma 2.3 that $H_{n}$ is a normal subgroup of $G$ with finite exponent and that $G_{n} / H_{n}$ is a residually finite non-abelian group. Thus there exist a finite non-abelian subgroup $K_{n+1}$ of $G_{n}$ and a normal subgroup of finite index $G_{n+1}$ of $G$ such that $\left\langle K_{1}, \ldots, K_{n}, K_{n+1}\right\rangle \cap G_{n+1} \leq H_{n}, K_{n+1} / H_{n} \cap K_{n+1}$ is nonabelian and $H_{n} \leq G_{n+1}<G_{n}$. The construction of the subgroups $K_{1}, \ldots, K_{n}, \ldots$ and $G_{0}, G_{1}, \ldots, G_{n}, \ldots$ is complete. 
Consider the subgroups

$$
L=\bigcup_{n \in \mathbb{N}} L_{n} \quad \text { and } \quad H=\bigcup_{n \in \mathbb{N}} H_{n} .
$$

Since $H_{n} \leq G_{n+1}$ for each $n$, we have that

$$
H \leq \bigcap_{m \in \mathbb{N}} G_{m} .
$$

In particular, $H \cap K_{n} \leq G_{n} \cap K_{n} \leq H_{n-1}$ and so the factor group $K_{n} /\left(H \cap K_{n}\right)$ is non-abelian for each $n$. It follows also that

$$
K_{n+1} H \cap L_{n} \leq G_{n} \cap L_{n} \leq H_{n-1} \leq H .
$$

Finally, if $m$ and $n$ are positive integers with $m<n$, then

$$
\left[K_{n}, K_{m}\right] \leq\left[G_{n-1}, K_{m}^{G}\right] \leq\left[G_{m}, K_{m}^{G}\right] \leq G_{m} \cap K_{m}^{G} \leq H .
$$

Therefore the factor group $L H / H$ is the direct product of its finite non-abelian subgroups $K_{1} H / H, K_{2} H / H, \ldots, K_{n} H / H, \ldots$ On the other hand, $L H / H$ is an $F C$ group with the property $Q F$, and hence it cannot be decomposed in such a product (see [4, Lemma 4.5]). This contradiction completes the proof.

LEMMA 3.7. Let $G$ be a residually finite metabelian p-group with the property $Q F$. Then $G$ is abelian-by-finite.

Proof. By Lemma 3.4, $G$ contains a normal subgroup $H$ of finite index such that the factor groups $\Omega_{n+1}(H) / \Omega_{n}(H)$ are abelian for all $n \geq 0$. Moreover, Lemma 3.2 allows us to suppose that the derived subgroup $H^{\prime}$ of $H$ has infinite exponent. Thus the normal closure $E^{H}$ of any finite subgroup $E$ of $H$ is central-by-finite by Lemma 3.3, and in particular $E^{H}$ has finite exponent. Lemma 3.6 yields that there exists in $H$ a subgroup $K$ of finite index whose derived subgroup $K^{\prime}$ has finite exponent. As $K$ is abelian-by-finite by Lemma 3.2, it follows that $G$ is itself abelian-by-finite.

Our next lemma proves that the theorem holds for soluble primary groups.

LEMMA 3.8. Let $G$ be a soluble p-group with the property $Q F$. Then $G$ is abelianby-finite.

PROOF. Let $K$ be the smallest non-trivial term of the derived series of $G$. By induction on the derived length of $G$ we may suppose that the factor group $G / K$ is abelian-by-finite, so that $G$ is metabelian-by-finite and hence without loss of generality it can be assumed that $G$ is metabelian. Let $J$ be the finite residual of the derived 
subgroup $G^{\prime}$ of $G$, and put $\bar{G}=G / J$. As $\bar{G}^{\prime}$ is residually finite, by Lemma 3.5 the factor group $\bar{G} / Z(\bar{G})$ is also residually finite, so that $\bar{G} / Z(\bar{G})$ is abelian-by-finite by Lemma 3.7 and $\bar{G}$ is nilpotent-by-finite. If $x$ is any element of $G$, the subgroup $\langle x\rangle G^{\prime}$ has the property $C F$ (see [4, Lemma 4.1]) and hence $x$ normalizes all subgroups of $J$ (see [1, Lemma 4.1]). Thus $G$ acts on $J$ as a group of power automorphisms, and in particular $G / C_{G}(J)$ is finite. As the subgroup $C_{G}(J)$ is nilpotent-by-finite, it follows that $G$ itself is nilpotent-by-finite and so it is abelian-by-finite (see [4, Lemma 4.2]).

LEMma 3.9. Let $G$ be a p-group with the property $Q F$ and let $F$ be the Fitting subgroup of $G$. Then the subgroup $F^{\prime \prime}$ is contained in the FC-centre of $G$. In particular, the Fitting subgroup of $G$ is soluble.

PROOF. Let $E$ be any finite subgroup of $F$. The normal closure $N=E^{G}$ of $E$ in $G$ is nilpotent, and so it has finite exponent. It follows that $N$ is abelian-by-finite and hence it is a $C F$-group (see [4, Lemma 4.3 and Lemma 4.1]). Thus $N$ contains an abelian normal subgroup of finite index $A$ such that all subgroups of $A$ are normal in $N$ (see [1, Lemma 2.1]). Let $L$ be a finite subgroup such that $N=A L$. Since $L$ induces on $A$ a group of power automorphisms, we have that $L / C_{L}(A)$ is abelian and so $N^{\prime} \leq A C_{L}(A)$. Therefore $N^{\prime \prime}$ is contained in $C_{L}(A)$, and in particular $N^{\prime \prime}$ is a finite normal subgroup of $G$ containing $E^{\prime \prime}$. It follows that $F^{\prime \prime}$ is contained in the $F C$-centre of $G$. As primary $F C$-groups with the property $Q F$ are soluble (see [4, Lemma 4.5]), we have also that $F$ is soluble.

We can now prove the main result of this section.

THEOREM 3.10. Let $G$ be a locally finite p-group with the property $Q F$. Then $G$ is abelian-by-finite.

PROOF. Let $n$ be a non-negative integer such that $\Omega_{n}(G)$ is soluble. Since $\Omega_{n+1}(G) / \Omega_{n}(G)$ is generated by elements of order $p$, all its permutable subgroups are normal. In particular, $\Omega_{n+1}(G) / \Omega_{n}(G)$ is a $C F$-group, so that it is abelian-by-finite (see [1]) and hence $\Omega_{n+1}(G)$ is also soluble. It follows now from Lemma 3.8 that each $\Omega_{n}(G)$ is abelian-by-finite. If $A$ is an abelian normal subgroup of finite index of $\Omega_{n}(G)$, then the normal closure $A^{G}$ is generated by finitely many conjugates of $A$ and so its centre $Z\left(A^{G}\right)$ has finite index in $\Omega_{n}(G)$. As $Z\left(A^{G}\right)$ is contained in the Fitting subgroup $F$ of $G$, the factor group $\Omega_{n}(G) F / F$ is finite for every $n$, so that $G / F$ is an $F C$-group, and hence it is soluble (see [4, Lemma 4.5]). Therefore $G$ is soluble by Lemma 3.9, and hence it is abelian-by-finite by Lemma 3.8. 


\section{Proof of the theorem}

The first result of this section deals with the case of periodic locally nilpotent groups.

LEMMA 4.1. Let $G$ be a periodic locally nilpotent $Q F$-group. Then $G$ contains $a$ quasihamiltonian subgroup of finite index.

Proof. By Theorem 3.10, the Sylow $p$-subgroup $G_{p}$ of $G$ is abelian-by-finite for each prime $p$. Let $\pi$ be the set of all prime numbers $p$ such that $G_{p}$ is not quasihamiltonian, and assume for a contradiction that $\pi$ is infinite. If $p \in \pi$, let $X_{p}$ be a non-permutable subgroup of $G_{p}$, and put

$$
X=\operatorname{Dr}_{p \in \pi} X_{p}
$$

As $G$ is a $Q F$-group, the permutable core $X_{*}$ of $X$ contains $X_{q}$ for some $q \in \pi$, and so $X_{q}=X_{*} \cap G_{q}$ is permutable in $G_{q}$. This contradiction shows that all but finitely many $G_{q}$ are quasihamiltonian, and hence $G$ is quasihamiltonian-by-finite.

LEMMA 4.2. Let $G$ be a periodic soluble $Q F$-group. If $G$ is countable, then it contains a quasihamiltonian subgroup of finite index.

PROOF. In the set $\pi=\pi(G)$ consider the relation $\sim$ defined by $p \sim q$ if and only if $p \neq q$ and each $p$-element of $G$ commutes with all $q$-elements of $G$. Let $\rho$ be any subset of $\pi$ and write $\rho^{\prime}=\pi \backslash \rho$. Since $G$ is countable, there exist a $\rho$-subgroup $H$ and a $\rho^{\prime}$-subgroup $K$ of $G$ such that $G=H K$ (see for instance [3, Lemma 5.1.6]). The normal closure $H_{*}^{G}$ of the permutable core $H_{*}$ of $H$ in $G$ is likewise a $\rho$-subgroup, and from

$$
H_{*}^{G} H=H\left(H_{*}^{G} H \cap K\right)=H
$$

it follows that $H_{*}^{G}=H_{*}$. Hence $H_{*}$ and similarly $K_{*}$ are normal subgroups of $G$, so that $H_{*} K_{*}=H_{*} \times K_{*}$. As $G$ is a $Q F$-group, the subgroup $H_{*} K_{*}$ has finite index in $G$. If $p_{1}$ and $p_{2}$ are prime numbers in $\rho$ and $\rho^{\prime}$, respectively, which do not divide the order of $G / H_{*} K_{*}$, it follows that $p_{1} \sim p_{2}$. If $\pi$ is infinite, then any infinite subset $\sigma$ of $\pi$ contains distinct elements $p$ and $q$ such that $p \sim q$, because we can always choose an infinite subset $\rho$ of $\sigma$ such that the intersection $\rho^{\prime} \cap \sigma$ is infinite. Application of Ramsey's theorem yields that in this case $\sigma$ contains an infinite subset $\tau$ such that $p \sim q$ for all distinct primes $p, q \in \tau$.

Let $\theta$ be the set of all prime numbers $q$ for which the Sylow $q$-subgroups of $G$ are not normal, and assume that $\theta$ is infinite. Then $\theta$ contains an infinite subset 
$\left\{q_{n} \mid n \in \mathbb{N}\right\}$ such that $q_{i} \sim q_{j}$ if $i \neq j$. For each positive integer $n$, let $Q_{n}$ be a Sylow $q_{n}$-subgroup of $G$, so that

$$
Q=\left\langle Q_{n} \mid n \in \mathbb{N}\right\rangle=\operatorname{Dr}_{n \in \mathbb{N}} Q_{n} .
$$

As the permutable core $Q_{*}$ of $Q$ has finite index in $Q$, there is a positive integer $m$ such that $Q_{m}$ is contained in $Q_{*}$. Then $Q_{m}$ is ascendant, and so even normal in $G$. This contradiction shows that the set $\theta$ is finite; in particular, if $R$ is the Hirsch-Plotkin radical of $G$, the set $\pi(G / R)$ is finite. Moreover, by Theorem 3.10 each non-trivial Sylow subgroup of $G$ contains a non-trivial permutable abelian subgroup of $G$, and hence all Sylow subgroups of $G / R$ are finite. It follows that $G / R$ is finite, so that by Lemma $4.1, G$ is quasihamiltonian-by-finite.

A group $G$ is called a $B Q F$-group if there exists a positive integer $k$ such that $\left|H / H_{*}\right| \leq k$ for all subgroups $H$ of $G$. It has been proved in [4] that the statement of the theorem is true in the case of $B Q F$-groups. As we will use this fact in the proof of our main theorem, we state it here as a lemma.

LEMMA 4.3. Let $G$ be a locally finite $B Q F$-group. Then $G$ contains a quasihamiltonian subgroup of finite index.

ProOF OF THE THEOREM. Suppose first that $G$ is soluble, and assume that it is not quasihamiltonian-by-finite, so that by Lemma 4.3 the property $B Q F$ does not hold for $G$. If $X$ is any subgroup of $G$, and $E$ is a finite subgroup such that $X=X_{*} E$, then

$$
\left|X: X_{*}\right|=\left|E: E \cap X_{*}\right| \leq\left|E: E_{*}\right| .
$$

It follows that there exists a sequence $\left(E_{n}\right)_{n \in \mathbb{N}}$ of finite subgroups of $G$ such that

$$
\left|E_{1}:\left(E_{1}\right)_{*}\right|<\left|E_{2}:\left(E_{2}\right)_{*}\right|<\cdots<\left|E_{n}:\left(E_{n}\right)_{*}\right|<\cdots .
$$

For each positive integer $n$ put $K_{n}=\left(E_{n}\right)_{*}$ and let $\left\{U_{n, 1} / K_{n}, \ldots, U_{n, r_{n}} / K_{n}\right\}$ be the set of all non-trivial subgroups of $E_{n} / K_{n}$. Clearly, there exist elements $g_{n, 1}, \ldots, g_{n, r_{n}}$ of $G$ such that $\left\langle g_{n, i}\right\rangle U_{n, i} \neq U_{n, i}\left\langle g_{n, i}\right\rangle$ for all $i=1, \ldots, r_{n}$. Consider the countable subgroup $H=\left\langle E_{n}, g_{n, 1}, \ldots, g_{n, r_{n}} \mid n \in \mathbb{N}\right\rangle$. Clearly, each $E_{n}$ has the same permutable core in $H$ and in $G$, so that in particular $H$ is not a $B Q F$-group. Moreover, $H$ contains a quasihamiltonian normal subgroup of finite index $L$ by Lemma 4.2. Let $\pi$ be the set of all prime numbers $p$ such that the unique Sylow $p$-subgroup $L_{p}$ of $L$ contains a subgroup $V_{p}$ which is not permutable in $G$, and put

$$
V=\operatorname{Dr}_{p \in \pi} V_{p} .
$$

If $\pi$ is infinite, there exists $p \in \pi$ such that $V_{p}$ is contained in the permutable core $V_{*}$ of $V$; then $V_{p}$ is a direct factor of $V_{*}$ and hence is permutable in $G$ (see [11, 
Lemma 6.2.16]). This contradiction shows that the set $\pi$ is finite. For each prime $p \in \pi(L), H / L_{p^{\prime}}$ is a finite extension of a primary subgroup, and so it is abelian-byfinite by Theorem 3.10. It follows that $H / L_{p^{\prime}}$ is a $C F$-group (see [4, Lemma 4.1]), and hence a $B C F$-group (see [1]), so that there exists a positive integer $k_{p}$ such that $\left|W L_{p^{\prime}}:\left(W L_{p^{\prime}}\right)_{H}\right| \leq k_{p}$ for every subgroup $W$ of $L_{p}$. Moreover, the unique Sylow $p$-subgroup $P$ of $\left(W L_{p^{\prime}}\right)_{H}$ is normal in $H$ and hence

$$
\left|W: W_{H}\right| \leq|W: P|=\left|W L_{p^{\prime}}:\left(W L_{p^{\prime}}\right)_{H}\right| \leq k_{p} .
$$

Put $\pi=\left\{p_{1}, \ldots, p_{t}\right\}, k=k_{p_{1}} \ldots k_{p_{t}},|H: L|=m$, and let $Y$ be any subgroup of $H$. Then

$$
Y \cap L=\left(Y \cap L_{p_{1}}\right) \times \cdots \times\left(Y \cap L_{p_{t}}\right) \times \operatorname{Dr}_{p \notin \pi}\left(Y \cap L_{p}\right)
$$

and the subgroup

$$
Z=\left(Y \cap L_{p_{1}}\right)_{H} \times \cdots \times\left(Y \cap L_{p_{l}}\right)_{H} \times \operatorname{Dr}_{p \notin \pi}\left(Y \cap L_{p}\right)
$$

is permutable in $H$. Since $|Y: Z|=|Y: Y \cap L| \cdot|Y \cap L: Z| \leq m k$, it follows that $H$ is a $B Q F$-group, and this contradiction proves the statement when $G$ is soluble.

Suppose now that $G$ is an arbitrary locally finite $Q F$-group. It follows from the Hall-Kulatilaka theorem that every infinite homomorphic image of $G$ contains an infinite abelian permutable subgroup, so that $G$ is radical-by-finite, and without loss of generality it can be assumed that $G$ is a radical group. Let $R_{1}$ be the HirschPlotkin radical of $G$ and $R_{2} / R_{1}$ the Hirsch-Plotkin radical of $G / R_{1}$. Then $R_{2}$ is soluble-by-finite by Lemma 4.1 , and it follows from the soluble case that $R_{2}$ contains a quasihamiltonian normal subgroup of finite index. Therefore $R_{2} / R_{1}$ is finite and so $G / R_{1}$ is also finite and hence $G$ is quasihamiltonian-by-finite.

\section{Acknowledgements}

We wish to thank the referee for his helpful comments. This work was done while the last author was visiting the University of Napoli Federico II. He thanks the "Dipartimento di Matematica e Applicazioni" for financial support.

\section{References}

[1] J. T. Buckley, J. C. Lennox, B. H. Neumann, H. Smith and J. Wiegold, 'Groups with all subgroups normal-by-finite', J. Aust. Math. Soc. Ser. A 59 (1995), 384-398.

[2] F. de Giovanni, C. Musella and Y. P. Sysak, 'Groups with almost modular subgroup lattice', $J$. Algebra 243 (2001), 738-764. 
[3] M. R. Dixon, Sylow theon; formations and fitting classes in locally finite groups, Series in Algebra 2 (World Scientific, Singapore, 1994).

[4] M. De Falco, F. de Giovanni and C. Musella, 'Groups in which every subgroup is permutable-byfinite', Comm. Algebra 32 (2004), 1007-1017.

[5] M. De Falco, F. de Giovanni, C. Musella and Y. P. Sysak, 'Groups in which every subgroup is nearly permutable'. Forum Math. 15 (2003), 665-677.

[6] K. Iwasawa, 'Über die endlichen Gruppen und die Verbände ihrer Untergruppen', J. Fac. Sci. Imp. Univ. Tokyo Sect. 14 (1941), 171-199.

[7] —., 'On the structure of infinite M-groups', Japan J. Math. 18 (1943), 709-728.

[8] W. Möhres, 'Hyperzentrale Torsiongruppen deren Untergruppen alle subnormal sind', Illinois $J$. Math. 35 (1991), 147-157.

[9] B. H. Neumann, 'Groups with finite classes of conjugate subgroups', Math. Z. 63 (1955), 76-96.

[10] D. J. S. Robinson, Finiteness conditions and generalized soluble groups (Springer, Berlin, 1972).

[11] R. Schmidt, Subgroup lattices of groups (de Gruyter, Berlin, 1994).

[12] S. E. Stonehewer, 'Permutable subgroups of infinite groups', Math. Z. 125 (1972), 1-16.

Dipartimento di Matematica e Applicazioni

Università di Napoli Federico II

Complesso Universitario Monte S. Angelo

Via Cintia

I-80126 Napoli

Italy

e-mail:mdefalco@unina.it

degiovan@unina.it

cmusella@unina.it
Institute of Mathematics

Ukrainian National Academy

of Sciences

vul. Tereshchenkivska 3

$01601 \mathrm{Kiev}$

Ukraine

e-mail: sysak@imath.kiev.ua 
